\title{
ON THE CONVERGENCE OF QUADRATURE FORMULAS RELATED TO AN INFINITE INTERVAL*
}

BY

J. V. USPENSKY

The quadrature formulas we shall discuss in this paper belong to the same type as the well known formula given by Gauss. They can be characterized as follows. Let $p(x)$ denote a function which does not assume negative values in a given interval $(a, b)$ and is subject to the condition that the integrals

$$
c_{m}=\int_{a}^{b} x^{m} p(x) d x
$$

or moments of the distribution determined by $p(x)$, exist. Under such circumstances it is always possible to find $n$ real numbers

$$
x_{1}<x_{2}<x_{3}<\cdots<x_{n}
$$

belonging to the interval $(a, b)$ together with $n$ corresponding constants $A_{1}, A_{2}, \cdots, A_{n}$ such that the formula

$$
\int_{a}^{b} p(x) f(x) d x=A_{1} f\left(x_{1}\right)+A_{2} f\left(x_{2}\right)+\cdots+A_{n} f\left(x_{n}\right)
$$

holds true whenever $f(x)$ is a polynomial whose degree does not exceed $2 n-1$, and this property completely determines the numbers $x_{1}, x_{2}, \cdots, x_{n}$ as well as the corresponding coefficients $A_{1}, A_{2}, \cdots, A_{n}$. It is important to notice that these coefficients are all positive. For any function $f(x)$ which is not a polynomial of degree $\leqq 2 n-1$ the right member of (1) ceases to represent exactly the integral in the left member, and we must add the remainder $R_{n}$ in order to have an exact equality:

$$
\int_{a}^{b} p(x) f(x) d x=A_{1} f\left(x_{1}\right)+A_{2} f\left(x_{2}\right)+\cdots+A_{n} f\left(x_{n}\right)+R_{n} .
$$

For any function $f(x)$ possessing a derivative of order $2 n$ the following expression for the remainder $R_{n}$ can be obtained:

$$
R_{n}=\frac{f^{(2 n)}(\xi)}{2 n !} \int_{a}^{b} p(x) \omega_{n}^{2}(x) d x
$$

* Presented to the Society, April 16, 1927; received by the editors in September, 1927. 
where

$$
\omega_{n}(x)=\left(x-x_{1}\right)\left(x-x_{2}\right) \cdots\left(x-x_{n}\right),
$$

and $\xi$ denotes a certain number belonging to the interval $(a, b)$. For a given particular function and for a given value of $\boldsymbol{n}$ this expression may be useful in that it enables us to form an idea of the accuracy attained by using the approximate formula (1). But it is hardly adapted to yield any conclusion as to the behavior of $R_{n}$ when $n$ increases indefinitely. Looking at the question from this point of view the problem naturally arises to investigate whether the approximate formula (1) can be made to furnish an indefinite approximation by choosing the number $n$, which is at our disposal, sufficiently large, or, in other words, whether the remainder $R_{n}$ in (2) converges to zero with indefinitely increasing $n$. This important problem of convergence of quadrature formulas of Gaussian type has been almost completely solved in case of the finite interval $(a, b)$ by Stieltjes in his beautiful paper Quelques recherches sur la théorie des quadratures dites mécaniques. * The case of an infinite interval is more difficult, and although we possess a very profound investigation concerning the special case

$$
f(x)=\frac{1}{z-x}
$$

by Stieltjes, $\nmid$ it seems that this problem has never been considered for a more general type of functions $f(x)$ except in an article by the author published in 1916.f

In the present paper we endeavor to treat the same problem by a totally different method, which permits us to reach more general conclusions than those established in the above mentioned article.

1. We begin with the case in which $b=+\infty$ while $a$ is any finite number, and, to avoid unnecessary complications, we suppose at first $a=0$. In this case all the numbers $x_{1}, x_{2}, \cdots, x_{n}$ are positive and furthermore the following important inequality holds:

$$
L_{m, n}=A_{1} x_{1}^{m}+A_{2} x_{2}^{m}+\cdots+A_{n} x_{n}^{m} \leqq c_{m} \quad(m=0,1,2, \cdots) .
$$

For taking $f(x)=x^{m}$ we see from (3) that $R_{n}=0$ when $m<2 n$, while $R_{n}>0$

\footnotetext{
* Annales de l'Ecole Normale Supérieure, (3), vol. 1 (1884).

$\dagger$ Recherches sur les fractions continues, Annales de la Faculté des Sciences de Toulouse, vol. 7 (1894).

$\ddagger$ On the convergence of quadrature formulas between infinite limits, Bulletin of the Russian Academy of Sciences, 1916 (in Russian).
} 
when $m \geqq 2 n$; that is, $L_{m, n}=c_{m}$ in the first case and $L_{m, n}<c_{m}$ in the second case.

From now on we shall assume that the function $p(x)$ satisfies the condition

$$
\int_{\alpha}^{\beta} p(x) d x>0 \quad \text { if } \quad 0 \leqq \alpha<\beta,
$$

and that constants $C$ and $R$ may be found such that

$$
\frac{c_{m}}{(2 m+1) !}<C R^{2 m}
$$

for $m=1,2,3, \cdots$.

These conditions being fulfilled, we can establish the convergence of (1) first for a special type of function $f(x)$, namely

$$
f(x)=e^{s x^{1 / 2}}+e^{-s x^{1 / 2}}
$$

denoting by $s$ a complex parameter. For such a choice of $f(x)$ the left member of (1) is

$$
\phi_{n}(s)=\sum_{k=1}^{n} A_{k}\left(e^{s x^{1 / 2}}+e^{-s x^{1 / 2}}\right)
$$

which, after substituting for exponential functions the corresponding power series, gives

$$
\phi_{n}(s)=2 \sum_{m=0}^{\infty} \frac{L_{m, n} s^{2 m}}{2 m !}
$$

Now,

$$
L_{m, n} \leqq c_{m}<C(2 m+1) ! R^{2 m}
$$

and the series

$$
\sum_{m=0}^{\infty}(2 m+1) s^{2 m} R^{2 m}
$$

is absolutely and uniformly convergent if

$$
|s| \leqq \rho<1 / R \text {. }
$$

The series (6) is therefore uniformly convergent with respect to $n$ and $s$ provided $s$ satisfies the inequality (7), and since

$$
L_{m, n} \rightarrow c_{m} \text { as } n \rightarrow \infty,
$$

it follows that

$$
\lim \phi_{n}(s)=2 \sum_{m=0}^{\infty} \frac{c_{m} s^{2 m}}{2 m !}
$$


uniformly in $s$. The sum of this series may be represented as a definite integral. This series being convergent for $s=a<1 / R$, where $a$ is a positive number, it follows easily that the integral

$$
\int_{0}^{\infty} p(x)\left(e^{a x^{1 / 2}}+e^{-a x^{1 / 2}}\right) d x
$$

exists, and furthermore that

$$
2 \sum_{m=0}^{\infty} \frac{c_{m} a^{2 m}}{2 m !}=\int_{0}^{\infty} p(x)\left(e^{a x^{1 / 2}}+e^{-a x^{1 / 2}}\right) d x .
$$

This being established, it is not difficult to prove that

$$
2 \sum_{m=0}^{\infty} \frac{c_{m} s^{2 m}}{2 m !}=\int_{0}^{\infty} p(x)\left(e^{s x^{1 / 2}}+e^{-s x^{1 / 2}}\right) d x,
$$

provided

$$
|s| \leqq a<1 / R
$$

That is,

$$
\lim \phi_{n}(s)=\int_{0}^{\infty} p(x)\left(e^{s x^{1 / 2}}+e^{-s x^{1 / 2}}\right) d x
$$

uniformly in $s$ under the same conditions. The integral in the right member is uniformly convergent if $0 \leqq R(s) \leqq a$, and therefore represents a regular analytic function in the region $0 \leqq R(s) \leqq a$, which we shall denote by $\phi(s)$.

Putting $s=\sigma+i \tau$, it is obvious that

$$
\left|\phi_{n}(s)\right| \leqq \phi_{n}(a)
$$

and if $0 \leqq \sigma \leqq a$

That is,

$$
\phi_{n}(\sigma) \leqq \phi_{n}(a) \leqq \sum_{m=0}^{\infty} \frac{c_{m} a^{2 m}}{2 m !}=\phi(a)
$$

$$
\left|\phi_{n}(s)\right| \leqq \phi(a)
$$

whatever $n$ may be if only $0 \leqq R(s) \leqq a$. In the finite region $0 \leqq \sigma \leqq a$, $-T \leqq \tau<T$, the sequence of analytic functions

$$
\phi_{1}(s), \quad \phi_{2}(s), \quad \phi_{3}(s), \cdots
$$

is therefore uniformly bounded and we have established that this sequence converges uniformly to the analytic function $\phi(s)$ in the region $|s| \leqq a$. By a known theorem due to Stieltjes this sequence is uniformly convergent to 
$\phi(s)$ in the entire region $0 \leqq \sigma \leqq a,-T \leqq \tau \leqq T$, that is, (8) holds true in this region.

2. Let $a$ represent again a positive number $<1 / R$ and let $\rho$ be any positive number. Starting from the well known formula

$$
\begin{aligned}
& \frac{1}{2 \pi i} \int_{a-i \infty}^{a+i \infty} \frac{e^{w o s} d s}{s^{1+\rho}}=\frac{w^{\rho}}{\Gamma(1+\rho)} \text { if } w>0, \\
& \frac{1}{2 \pi i} \int_{a-i \infty}^{a+i \infty} \frac{e^{w o s} d s}{s^{1+\rho}}=0 \text { if } w \leqq 0,
\end{aligned}
$$

we find that

$$
\frac{1}{2 \pi i} \int_{a-i \infty}^{a+i \infty} \frac{e^{-s t} \phi_{n}(s)}{s^{1+\rho}} d s=\frac{1}{\Gamma(1+\rho)} \sum_{x_{k} \geq t^{2}} A_{k}\left(x_{k}^{1 / 2}-t\right)^{\rho}
$$

for any positive number $t$, the sum being extended over all $x_{k}$ satisfying the inequality $x_{k} \geqq t^{2}$. We have also

$$
\frac{1}{2 \pi i} \int_{a-i \infty}^{a+i \infty} \frac{e^{-s t} \phi(s)}{s^{1+\rho}} d s=\frac{1}{\Gamma(1+\rho)} \int_{t^{2}}^{\infty} p(x)\left(x^{1 / 2}-t\right)^{\rho} d x .
$$

From the fact that

$$
\left|\phi_{n}(a+i \tau)\right| \leqq \phi(a)
$$

for every $n$, and that $\phi_{n}(a+i \tau)$ converges uniformly to $\phi(a+i \tau)$ in any finite interval $-T \leqq \tau \leqq T$, we can easily infer that

$$
\lim _{n=\infty} \int_{a-i \infty}^{a+i \infty} \frac{e^{-s t} \phi_{n}(s)}{s^{1+\rho}} d s=\int_{a-i \infty}^{a+i \infty} \frac{e^{-s t} \phi(s)}{s^{1+\rho}} d s,
$$

which by (9) and (10) is equivalent to

$$
\lim \sum_{x_{k} \geq t^{2}} A_{k}\left(x_{k}^{1 / 2}-t\right)^{\rho}=\int_{t^{2}}^{\infty} p(x)\left(x^{1 / 2}-t\right)^{\rho} d x,
$$

or, putting $t^{2}=\alpha$,

$$
\lim \sum_{x_{k} \geq \alpha} A_{k}\left(x_{k}^{1 / 2}-\alpha^{1 / 2}\right)^{\rho}=\int_{\alpha}^{\infty} p(x)\left(x^{1 / 2}-\alpha^{1 / 2}\right)^{\rho} d x .
$$

Starting from this important result, we shall prove that

$$
\lim \sum_{x_{k} \geq \alpha} A_{k}=\int_{\alpha}^{\infty} p(x) d x .
$$


Let $\epsilon$ denote any given positive number however small. We choose first a positive number $\delta$ so small and another positive number $L$ so large that

$$
\begin{gathered}
\int_{L}^{\infty} p(x) x^{1 / 2} d x<\epsilon, \\
\frac{1}{1+\delta} \int_{0}^{\alpha} p(x) d x-\frac{2 \epsilon}{1+\delta}>\int_{0}^{\alpha} p(x) d x-3 \epsilon, \\
\frac{1}{1-\delta} \int_{0}^{\alpha} p(x) d x+\frac{2 \epsilon}{1-\delta}<\int_{0}^{\alpha} p(x) d x+3 \epsilon .
\end{gathered}
$$

The integrals

$$
\int_{\alpha \rightarrow \eta}^{\infty} p(x)\left(x^{1 / 2}-(\alpha-\eta)^{1 / 2}\right)^{\rho} d x \text { and } \int_{\alpha}^{\infty} p(x)\left(x^{1 / 2}-\alpha^{1 / 2}\right)^{\rho} d x
$$

being continuous functions of $\eta$ and $\rho$ in the region $\eta>0, \rho>0$, we can take $\eta$ and $\sigma$ so small that

$$
\begin{aligned}
& \int_{\alpha-\eta}^{\infty} p(x)\left(x^{1 / 2}-(\alpha-\eta \eta)^{1 / 2}\right)^{\rho} d x<\int_{\alpha}^{\infty} p(x) d x+\epsilon, \\
& \int_{\alpha}^{\infty} p(x)\left(x^{1 / 2}-\alpha^{1 / 2}\right)^{\rho} d x>\int_{\alpha}^{\infty} p(x) d x-\epsilon,
\end{aligned}
$$

whenever $0<\rho \leqq \sigma$. Having thus determined $L, \delta, \eta$, we take $\rho$ so small as to satisfy the inequalities

$$
\left(\alpha^{1 / 2}-(\alpha-\eta)^{1 / 2}\right)^{\rho}>1-\delta,(4 L)^{\rho / 2}<1+\delta, \quad \rho \leqq \sigma, \rho \leqq 1 .
$$

Finally we can take $N$ so large as to have

$$
\begin{aligned}
& \text { (18) } \sum_{x_{k} \geq \alpha-1} A_{k}\left(x_{k}{ }^{1 / 2}-(\alpha-\eta)^{1 / 2}\right)^{\rho}<\int_{\alpha-\eta}^{\infty} p(x)\left(x^{1 / 2}-(\alpha-\eta)^{1 / 2}\right) \rho d x+\epsilon, \\
& \text { (19) } \sum_{x_{k} \geq \alpha} A_{k}\left(x_{k}^{1 / 2}-\alpha^{1 / 2}\right)^{\rho}>\int_{\alpha}^{\infty} p(x)\left(x^{1 / 2}-\alpha^{1 / 2}\right) \rho d x-\epsilon, \\
& \text { (20) } \\
& \sum_{x_{k} \geq L} A_{k}\left(x_{k}^{1 / 2}-L^{1 / 2}\right)^{\rho}<\int_{L}^{\infty} p(x)\left(x^{1 / 2}-L^{1 / 2}\right) \rho d x+\epsilon,
\end{aligned}
$$

for all $n>N$. Now

$$
\begin{aligned}
\sum_{x_{k} \geq \alpha-1} A_{k}\left(x_{k}^{1 / 2}-(\alpha-\eta)^{1 / 2}\right) \rho & \geqq \sum_{x_{k} \geq \alpha} A_{k}\left(x_{k}^{1 / 2}-(\alpha-\eta)^{1 / 2}\right)^{\rho} \\
& \geqq\left(\alpha^{1 / 2}-(\alpha-\eta)^{1 / 2}\right) \rho \sum_{x_{k} \geq \alpha} A_{k} \geqq(1-\delta) \sum_{x_{k} \geq \alpha} A_{k},
\end{aligned}
$$


therefore by (18), (16) and (15)

$$
\sum_{x_{k} \geqq \alpha} A_{k}<\int_{\alpha}^{\infty} p(x) d x+3 \epsilon .
$$

From (20) and (13) we conclude that

$$
\sum_{x_{k} \geqq 4 L} A_{k}\left(x_{k}^{1 / 2}-L^{1 / 2}\right)^{\rho}<2 \epsilon,
$$

and for $x_{k} \geqq 4 L$ we have

therefore

$$
\frac{x_{k}^{1 / 2}-\alpha^{1 / 2}}{x_{k}^{1 / 2}-L^{1 / 2}}<2
$$

$$
\sum_{x_{k} \geqq 4 L} A_{k}\left(x_{k}^{1 / 2}-\alpha^{1 / 2}\right)^{\rho}<2^{\rho} \cdot 2 \epsilon<(1+\delta) 2 \epsilon .
$$

This being established, from (19) and (17) we derive

$$
\sum_{\alpha \leqq x_{k} \leqq 4 L} A_{k}\left(x_{k}^{1 / 2}-\alpha^{1 / 2}\right)^{\rho}>\int_{\alpha}^{\infty} p(x) d x-2 \epsilon-2 \epsilon(1+\delta) ;
$$

but

$$
\sum_{\alpha \leqq x_{k} \leqq 4 L} A_{k}\left(x_{k}^{1 / 2}-\alpha^{1 / 2}\right)^{\rho}<(4 L)^{\rho / 2} \sum_{x_{k} \geqq \alpha} A_{k}<(1+\delta) \sum_{x_{k} \geqq \alpha} A_{k}
$$

and therefore

$$
\sum_{x_{k} \geqq \alpha} A_{k}>\int_{\alpha}^{\infty} p(x) d x-5 \epsilon .
$$

The inequalities (21) and (22) being satisfied, however small $\epsilon$ may be, (12) can be considered as established. It follows from (12) that

$$
\lim \sum_{\alpha \leqq x_{k} \leqq \beta} A_{k}=\int_{\alpha}^{\beta} p(x) d x>0
$$

whence we conclude: for all sufficiently large $n$ there are numbers $x_{k}$ falling in any given interval $(\alpha, \beta)$ provided the condition (5) is satisfied.

3. After this important conclusion has been reached, we can apply exactly the same reasoning that Stieltjes uses in an analogous case. Let $f(x)$ denote an integrable function in a finite interval $(0, G)$ and let us suppose $f(x)=0$ for $x>G$. We shall prove that the formula (1) is convergent for any such function. To this end we introduce a new variable

$$
y=\int_{0}^{x} p(x) d x
$$


increasing with increasing $x$ from 0 to $H=\int_{0}^{G} p(x) d x$. The values of this new variable corresponding to $x=x_{k}$ will be denoted by $y_{k}$. We shall also introduce the notation

$$
f(x)=\phi(y) .
$$

The following important inequalities due to Tchebycheff, ${ }^{*}$

show that

$$
\begin{aligned}
& A_{1}+A_{2}+\cdots+A_{k}>\int_{0}^{x_{k}} p(x) d x, \\
& A_{1}+A_{2}+\cdots+A_{k}<\int_{0}^{x_{k+1}} p(x) d x
\end{aligned}
$$

$$
0 \leqq y_{1}<A_{1}<y_{2}<A_{1}+A_{2}<y_{3}<A_{1}+A_{2}+A_{3}<\cdots .
$$

The number $l$ being determined by the condition

$$
A_{1}+A_{2}+\cdots+A_{l-1} \leqq H<A_{1}+A_{2}+\cdots+A_{l},
$$

we conclude easily from (23) that the sum

$$
S=A_{1} \phi\left(y_{1}\right)+A_{2} \phi\left(y_{2}\right)+\cdots+A_{l_{1}} \phi\left(y_{l-1}\right)
$$

converges to the limit

$$
\int_{0}^{H} \phi(y) d y
$$

with indefinitely increasing $n$ provided all $A_{k}$ approach 0 , which is the case, as may be easily seen from the inequality

$$
A_{k}<\int_{x_{k-1}}^{x_{k+1}} p(x) d x
$$

combined with the final conclusion in $\$ 2$. But the sum

$$
A_{1} f\left(x_{1}\right)+A_{2} f\left(x_{2}\right)+\cdots+A_{n} f\left(x_{n}\right)
$$

can differ from $S$ only by the term $A_{l} \phi\left(y_{l}\right)=A_{l} f\left(x_{l}\right)$ which converges to 0 , that is

$$
\lim \left(A_{1} f\left(x_{1}\right)+A_{2} f\left(x_{2}\right)+\cdots+A_{n} f\left(x_{n}\right)\right)=\int_{0}^{H} \phi(y) d y=\int_{0}^{G} p(x) f(x) d x
$$

as was stated.

* Tchebycheff, Sur les valeurs limites des intégrales, Journal de Mathématiques, 1874. 
4. Now we can prove that the convergence of (1) is assured for any integrable function which for sufficiently large $x$ satisfies the inequality

$$
|f(x)|<x^{m}
$$

where $m$ is a certain integer. Let us denote by $\epsilon$ a positive number taken arbitrarily and let us determine $G$ by the condition

$$
\int_{\sigma}^{\infty} p(x) x^{m} d x<\epsilon .
$$

Let $F(x)$ be defined as follows:

$$
\begin{aligned}
& F(x)=f(x) \quad \text { for } \quad 0 \leqq x \leqq G, \\
& F(x)=0 \text { for } x>G .
\end{aligned}
$$

We have, by the results of $\S 3$,

or

$$
\lim \sum_{k=1}^{n} A_{k} F\left(x_{k}\right)=\int_{0}^{\infty} p(x) F(x) d x,
$$

$$
\lim \sum_{x_{k} \leq G} A_{k} f\left(x_{k}\right)=\int_{0}^{G} p(x) f(x) d x .
$$

In particular

$$
\lim \sum_{x_{k} \leq G} A_{k} x_{k}^{m}=\int_{0}^{G} p(x) x^{m} d x,
$$

and as

$$
\sum_{k=1}^{n} A_{k} x^{m}=\int_{0}^{\infty} p(x) x^{m} d x,
$$

we get

that is

$$
\lim \sum_{x_{k}>G} A_{k} x_{k}^{m}=\int_{G}^{\infty} p(x) x^{m} d x<\epsilon,
$$

$$
\sum_{x_{k}>G} A_{k} x_{k}^{m}<2 \epsilon
$$

for all sufficiently large $n$, and a fortiori,

$$
\left|\sum_{x_{k}>G} A_{k} f\left(x_{k}\right)\right|<2 \epsilon .
$$

Now, for sufficiently large $n$,

$$
\left|\sum_{x_{k} \leq \theta} A_{k} f\left(x_{k}\right)-\int_{0}^{\infty} p(x) f(x) d x\right|<\epsilon,
$$


and therefore

$$
\left|\sum_{k=1}^{n} A_{k} f\left(x_{k}\right)-\int_{0}^{\infty} p(x) f(x) d x\right|<3 \epsilon
$$

for all sufficiently large $n$; that is,

$$
\lim \sum_{k=1}^{n} A_{k} f\left(x_{k}\right)=\int_{0}^{\infty} p(x) f(x) d x
$$

5. Now we can take the last step and prove the convergence of (1) for for any integrable function satisfying for sufficiently large $x$ the inequality

$$
|f(x)|<\frac{e^{r x^{1 / 2}}}{x^{1+p}}
$$

where $\rho$ is any positive number $<1$ and

$$
r=1 / R,
$$

$R$ being the same number as in (5)

For this purpose we shall first establish a certain inequality. If $a_{k}, b_{k}(k=1,2,3, \cdots)$ denote positive numbers, we have

$$
\sum_{1}^{n} a_{k} b_{k} \leqq\left(\sum_{1}^{n} a_{k}^{\sigma}\right)^{1 / \rho}\left(\sum_{1}^{n} b_{k}^{\sigma /(\sigma-1)}\right)^{1-1 / \sigma}
$$

provided $\sigma>1$. Here we take

$$
\sigma=1 / \rho, a_{k}=A_{k}^{p} x_{k}^{(m-1) \rho}, b_{k}=A_{k}^{1-\rho} x_{k}^{m(1-\rho)},
$$

which gives

whence

$$
\sum_{1}^{n} A_{k} x_{k}^{m-\rho} \leqq\left(\sum_{1}^{n} A_{k} x_{k}^{m-1}\right)^{\rho}\left(\sum_{1}^{n} A_{k} x_{k}{ }^{m}\right)^{1-\rho},
$$

$$
\sum_{1}^{n} A_{k} x_{k}^{m-\rho} \leqq c_{m-1} c_{m}^{1-p}
$$

But by (5),

$$
\begin{aligned}
c_{m-1} & <C(2 m-1) ! R^{2 m-2}, \\
c_{m} & <C(2 m+1) ! R^{2 m}
\end{aligned}
$$

from which, with (24),

$$
\sum_{1}^{n} A_{k} x_{k}^{m-\rho}<C R^{2 \dot{m}-2 \rho}(2 m-1)[2 m(2 m+1)]^{1-p},
$$


which is the required inequality. Now we consider the function

$$
f(x)=\frac{e^{r x^{1 / 2}}+e^{-r x^{1 / 2}}}{1+x^{1+\rho}},
$$

and form the sum

$$
S_{n}=\sum_{1}^{n} A_{k} f\left(x_{k}\right)=\sum_{1}^{n} A_{k} \frac{e^{r x_{k}^{1 / 2}}+e^{-r x_{k}{ }^{1 / 2}}}{1+x_{k}^{1+\rho}},
$$

which, putting

$$
\gamma_{m}=\sum_{1}^{n} \frac{A_{k} x_{k}^{m}}{1+x_{k}^{1+\rho}},
$$

can be represented by the series

As

$$
S_{n}=2 \sum_{m=0}^{\infty} \frac{\gamma_{m}}{2 m !} r^{2 m}
$$

$$
\gamma_{m}<\sum_{1}^{n} A_{k} x_{k}^{m-1-p}
$$

it follows from (25) that

$$
\frac{\gamma_{m}}{2 m !} r^{2 m}<\frac{C}{R^{2 \rho+2}} \frac{1}{2 m[(2 m-2)(2 m-1)]^{\rho}},
$$

which shows that the series converges uniformly in $n$.

By what has been established in $\$ 4$ we have

$$
\lim \gamma_{m}=\int_{0}^{\infty} \frac{p(x) x^{m}}{1+x^{1+\rho}} d x,
$$

and therefore

$$
\lim S_{n}=\int_{0}^{\infty} p(x) \frac{e^{r x^{1 / 2}}+e^{-r x^{1 / 2}}}{1+x^{1+\rho}} d x .
$$

We proceed now in the same way as in $\$ 4$. For any given $\epsilon>0$, a sufficiently large number $G$ is determined by the condition

$$
\int_{G}^{\infty} p(x) \frac{e^{r x^{1 / 2}}+e^{-r x^{1 / 2}}}{1+x^{1+\rho}} d x<\epsilon .
$$

Applying then the result established in $\S 3$, we have

$$
\lim \sum_{x_{k} \leq G} A_{k} \frac{e^{r x_{k}^{1 / 2}}+e^{-r x k^{1 / 2}}}{1+x_{k}^{1+\rho}}=\int_{0}^{G} p(x) \frac{e^{r x^{1 / 2}}+e^{-r x^{1 / 2}}}{1+x^{1+\rho}} d x,
$$


and therefore

$$
\sum_{x_{k}>G} A_{k} \frac{e^{r x_{k}^{1 / 2}}+e^{-r x_{k} / 2}}{1+x_{k}^{1+\rho}}<2 \epsilon
$$

for sufficiently large $n$. By hypothesis

$$
|f(x)|<\frac{e^{r x^{1 / 2}}}{x^{1+\rho}}<2 \frac{e^{r x^{1 / 2}}+e^{-r x^{1 / 2}}}{1+x^{1+\rho}}
$$

as soon as $x$ exceeds a certain finite limit, therefore

$$
\left|\sum_{x_{k}>G} A_{k} f\left(x_{k}\right)\right|<4 \epsilon
$$

for sufficiently large $n$. On the other hand

$$
\left|\sum_{x_{k} \leqq G} A_{k} f\left(x_{k}\right)-\int_{0}^{G} p(x) f(x) d x\right|<\epsilon
$$

for large $n$, from which, with the preceding, it follows that

$$
\left|\sum_{k=1}^{n} A_{k} f\left(x_{k}\right)-\int_{0}^{\infty} p(x) f(x) d x\right|<6 \epsilon
$$

for all sufficiently large $n$. The convergence of (1) for all functions satisfying the condition

is now completely proved.

$$
|f(x)|<\frac{e^{r x^{1 / 2}}}{x^{1+\rho}}
$$

6. Suppose we deal with the infinite interval $(a,+\infty)$ where $a$ is any finite number, so that we have

$$
\int_{a}^{\infty} p(x) f(x) d x=A_{1} f\left(x_{1}\right)+A_{2} f\left(x_{2}\right)+\cdots+A_{n} f\left(x_{n}\right)
$$

whenever $f(x)$ is a polynomial of degree $\leqq 2 n-1$. Putting

$$
p_{1}(t)=p(a+t) ; \quad t_{k}=x_{k}-a
$$

we have

$$
\int_{0}^{\infty} p_{1}(t) \phi(t) d t=A_{1} \phi\left(t_{1}\right)+A_{2} \phi\left(t_{2}\right)+\cdots+A_{n} \phi\left(t_{n}\right)
$$

for any polynomial $\phi(t)$ whose degree does not exceed $2 n-1$. According to what has been established, the last formula converges whenever the moments of $p_{1}(t)$ 


$$
\gamma_{m}=\int_{0}^{\infty} p_{1}(t) t^{m} d t
$$

satisfy an inequality of the form

$$
\gamma_{m}<D(2 m+1) ! R^{2 m}
$$

with certain constants $D$ and $R$, for any function $\phi(t)$ integrable in any finite interval and for large values of $t$ satisfying the condition

$$
|\phi(t)|<\frac{e^{r t^{1 / 2}}}{t^{1+\rho}}
$$

where $r=1 / R$ and $\rho$ is any positive number $<1$. We have

$$
\gamma_{m}=\int_{a}^{\infty} p(x)(x-a)^{m} d x
$$

so that for $a>0$

$$
\gamma_{m}<\int_{a}^{\infty} p(x) x^{m} d x<c_{m}
$$

while for $a<0$

$$
\gamma_{m}<c_{0}|a|^{m}+c_{m}+\frac{m}{1} c_{m-1}|a|+\frac{m(m-1)}{1 \cdot 2} c_{m-2}|a|^{2}+\cdots,
$$

and, if the condition

$$
c_{m}<C(2 m+1) ! R^{2 m}
$$

is satisfied,

$$
\begin{aligned}
\gamma_{m}<c_{0}|a|^{m} & +C(2 m+1) ! R^{2 m}\left[1+\frac{m}{1 \cdot 2 m(2 m+1)} \frac{|a|}{R^{2}}\right. \\
& \left.+\frac{m(m-1)}{1 \cdot 2 \cdot(2 m-2)(2 m-1) 2 m(2 m+1)}\left(\frac{|a|}{R^{2}}\right)^{2}+\cdots\right],
\end{aligned}
$$

whence

$$
\gamma_{m}<\left[c_{0}|a|^{m}+C e^{|a| / R^{2}}(2 m+1) ! R^{2 m}<2 C e^{|a| / R^{2}}(2 m+1) ! R^{2 m}\right.
$$

for all sufficiently large $m$. In every case (26) holds with

$$
D=2 C e^{|a| / R^{2}}
$$

and we can draw the following final conclusion: 
The quadrature formula related to the interval $(a, \infty)$ converges whenever the moments satisfy the condition

$$
c_{m}<C(2 m+1) ! R^{2 m}
$$

with certain constants $C$ and $R$, for any integrable function satisfying the inequality (for sufficiently large $x$ )

$$
|f(x)|<\frac{e^{r x^{1 / 2}}}{x^{1+\rho}}
$$

where $r=1 / R$ and $\rho$ is any positive number $<1$.

Taking

$$
f(x)=\frac{1}{z-x}
$$

with any complex $z$ not belonging to the interval $(a,+\infty)$, we see that the continued fraction

$$
\int_{a}^{\infty} \frac{p(x) d x}{z-x}=\frac{\lambda_{0}}{z-\alpha_{0}}-\frac{\lambda_{1}}{z-\alpha_{1}}-
$$

converges and represents the integral if only the moments $c_{m}$ satisfy the above mentioned condition.

To show how far-reaching this criterion is, let us take $a=0$ and $p(x)=e^{-x^{1 / 2}}$.

In this case $c_{m}=(2 m+1)$ !, so that $R=1, C=1$. The quadrature formula converges for any function satisfying the inequality

$$
|f(x)|<\frac{e^{x^{1 / n}}}{x^{1+\rho}}
$$

for large $x$ and, in particular, the continued fraction converges and represents the integral. We obtain the same result for

$$
p(x)=e^{-x^{\lambda}}
$$

with $\lambda>\frac{1}{2}$ because the $c_{m}$ corresponding to this $p(x)$ are obviously less than in the preceding case. But the convergence ceases with $\lambda=\frac{1}{2}$, for it is known that for $\lambda<\frac{1}{2}$ the problem of moments becomes indeterminate.

7. It remains to consider the case of an infinite interval $(-\infty, \infty)$. The analysis of this case is but slightly different, and we can confine ourselves to a very brief outline. We retain the fundamental assumption that

$$
\int_{\alpha}^{\beta} p(x) d x>0
$$


for any two numbers $\alpha, \beta$ satisfying the condition $\alpha<\beta$. As to the moments, we consider only those of even order and assume the existence of two constants $C$ and $R$ such that

$$
c_{2 m}<C(2 m) ! R^{2 m} .
$$

For moments of odd order we have

$$
\left|c_{2 m+1}\right| \leqq\left(c_{2 m} c_{2 m+2}\right)^{1 / 2}<C(2 m+1) ! R^{2 m+1}\left(\frac{2 m+2}{2 m+1}\right)^{1 / 2}<2 C(2 m+1) ! R^{2 m+1} .
$$

By means of these inequalities it can be shown in exactly the same way as in $\$ 1$ that the analytic function

$$
\phi_{n}(s)=\sum_{k=1}^{n} A_{k} e^{-s x_{k}}
$$

converges uniformly in $s$ to

provided

$$
\phi(s)=\int_{-\infty}^{\infty} p(x) e^{-s x} d x
$$

$$
|s| \leqq \rho<1 / R .
$$

The function $\phi(s)$ is a regular analytic function in the region $-a \leqq R(s) \leqq a$ where $0<a<1 / R$.

In the same region the sequence

$$
\left.\phi_{1}(s), \quad \phi_{2}(s), \phi_{3} s\right), \cdots
$$

is uniformly bounded and by Stieltjes' theorem $\phi_{n}(s)$ converges to $\phi(s)$ uniformly in a finite region

$$
-a \leqq \sigma \leqq a,-T \leqq \tau \leqq T .
$$

This being established, we reach in the same way as in $\$ 2$ the important result

$$
\lim \sum_{x_{k} \leqq t} A_{k}\left(t-x_{k}\right)^{\rho}=\int_{-\infty}^{t} p(x)(t-x)^{\rho} d x
$$

for every $\rho>0$, whence we can conclude that

and

$$
\lim \sum_{x_{k} \leqq \alpha} A_{k}=\int_{-\infty}^{\alpha} p(x) d x,
$$

$$
\lim \sum_{\alpha<x_{k} \leq \beta} A_{k}=\int_{\alpha}^{\beta} p(x) d x>0 .
$$


This shows that for sufficiently large $n$ there always numbers $x_{k}$ belonging to any given interval $(\alpha, \beta)$.

Again repeating the same reasoning as in $\$ 3$ we reach the conclusion that formula (1) converges for any integrable function which is zero outside a given finite interval. Then, as in $\$ 4$, this conclusion is extended to any function integrable in any finite interval and for sufficiently large $x$ satisfying the inequality

$$
|f(x)|<x^{m}
$$

Finally, the same conclusion may be extended to functions which for large $x$ satisfy the inequality

$$
|f(x)|<\frac{e^{r|x|}}{|x|^{1+p}}
$$

where $r=1 / R$ and $\rho$ is a positive number $<1$.

To prove this statement we observe that, the convergence being once established for a positive function $F(x)$, it is secured for any function satisfying the condition

$$
|f(x)|<F(x)
$$

at least for sufficiently large $x$. This may be easily proved by the same reasoning as developed in $\$ 4$. We take

$$
F(x)=\frac{e^{r x}+e^{-r x}}{1+|x|^{1+\rho}}
$$

where $0<\rho<1$, and obtain

$$
S=\sum_{1}^{n} A_{k} F\left(x_{k}\right)=2 \sum_{m=0}^{\infty} \frac{\gamma_{m}}{2 m !} r^{2 m},
$$

$\gamma_{m}$ being defined by

$$
\gamma_{m}=\sum_{1}^{n} A_{k} \frac{x_{k}{ }^{2 m}}{1+\left|x_{k}\right|^{1+\rho}}
$$

We have obviously

$$
\gamma_{m}<\sum_{1}^{n} A_{k}\left|x_{k}\right|^{2 m-1-p},
$$

but

$$
\sum_{1}^{n} A_{k}\left|x_{k}\right|^{2 m-1-\rho} \leqq\left(\sum_{1}^{n} A_{k} x_{k}^{2 m-2}\right)^{p}\left(\sum_{1}^{n} A_{k}\left|x_{k}\right|^{2 m-1}\right)^{1-p}
$$


or

$$
\sum_{1}^{n} A_{k}\left|x_{k}\right|^{2 m-1-\rho} \leqq 2 C R^{2 m-1-\rho} \frac{(2 m-1) !}{(2 m-1)^{\rho}} .
$$

The series

$$
\sum_{m=0}^{\infty} \frac{\gamma_{m}}{2 m !} r^{2 m}
$$

is therefore uniformly convergent in $n$, and as

we get

$$
\lim \gamma_{m}=\int_{-\infty}^{\infty} p(x) \frac{x^{2 m}}{1+|x|^{1+p}} d x,
$$

$$
\lim S=\int_{-\infty}^{\infty} p(x) \frac{e^{r x}+e^{-r x}}{1+|x|^{1+p}} d x
$$

so that the convergence is established for $F(x)$.

Now if

$$
|f(x)|<\frac{e^{r|x|}}{|x|^{1+\rho}}
$$

for sufficiently large $x$, it is obvious that under the same condition

$$
|f(x)|<2 F(x)
$$

which proves our statement.

8. In cases in which the rapidity of increase of the moments is less than was assumed, the class of functions for which the convergence is secured may be extended. Let us take for example $p(x)=e^{-x^{2}}$ and the interval $(-\infty, \infty)$. In this case

$$
c_{2 m}=\pi^{1 / 2} \frac{2 m !}{2^{2 m} m !} .
$$

Taking

we have

$$
F(x)=\frac{e^{x^{2}}}{1+|x|^{1+\rho}}, 1>\rho>0,
$$

where

$$
S=\sum_{1}^{n} A_{k} F\left(x_{k}\right)=\sum_{m=0}^{\infty} \frac{\gamma_{m}}{m !},
$$

$$
\gamma_{m}=\sum_{1}^{n} A_{k} \frac{x_{k}^{2 m}}{1+\left|x_{k}\right|^{1+\rho}}
$$


As

and

$$
\gamma_{m}<\sum_{1}^{n} A_{k}|x|^{2 m-1-\rho}
$$

$$
\begin{aligned}
\sum_{1}^{n} A_{k}\left|x_{k}\right|^{2 m-1-\rho} & \leqq\left(\sum_{1}^{n} A_{k} x_{k}{ }^{2 m-2}\right)^{\rho}\left(\sum_{1}^{n} A_{k}\left|x_{k}\right|^{2 m-1}\right)^{1-\rho} \\
& <\frac{\pi^{1 / 2} \cdot 2^{(1+\rho) / 2}}{2^{2 m-1}} \frac{(2 m-2) !}{(m-1) !}(2 m-1)^{(1-\rho) / 2},
\end{aligned}
$$

it is easy to see that the series representing $S$ converges uniformly in $n$ and therefore

$$
\lim S=\int_{-\infty}^{\infty} \frac{e^{x^{2}}}{1+|x|^{1+\rho}} d x .
$$

That is, the quadrature formula corresponding to $p(x)=e^{-x^{2}}$ converges for $F(x)$ and a fortiori it will be convergent for any function satisfying the condition

$$
|f(x)|<\frac{e^{x^{4}}}{|x|^{1+\rho}}, \quad \rho>0,
$$

for sufficiently large $x$

Another example of the same kind is given by the formula corresponding to $p(x)=e^{-x} x^{\alpha}$ and the interval $(0, \infty)$. In this case the convergence is secured for any integrable function satisfying the condition

$$
|f(x)|<\frac{e^{x}}{x^{\alpha+1+\rho}}, \quad \rho>0,
$$

for sufficiently large $x$.

Carueton College,

NORTHFIELD, MINN. 